\title{
Atención a los niños y las niñas víctimas de la violencia de género
}

\section{Atention to boys and girls victims of gender violence}

Pepa HORNO GOICOECHEA*

Fecha de Recepción: 18-09-2006

Fecha de Aceptación: 09-11-2006

\section{RESUMEN}

Save the Children desarrolló la investigación "Atención a niños y niñas víctimas de la violencia de género" que pretende analizar la atención que se está dando a los hijos e hijas de las mujeres víctimas de la violencia de género en el sistema de protección a la mujer. Los resultados de la investigación indican que los profesionales del ámbito social, policial y judicial entienden que los niños y niñas son víctimas de la violencia de género, son víctimas de maltrato psicológico no sólo por recibir agresiones fisicas también o por ser testigos directos de la violencia sino por vivir en un entorno donde se da esta violencia. Sin embargo, la investigación también denuncia que el sistema de protección a la mujer no ha contemplado las medidas específicas suficientes y necesarias para atender las necesidades diferenciales de estas víctimas ni cauces de participación de los niños y niñas en la toma de decisiones que les afectan directamente.

\section{PALABRAS CLAVE}

Niños y niñas, Violencia de género, Atención, Víctimas.

\section{ABSTRACT}

Save the Children developed the research "Attention to children victims of gender violence" whose objective is to analyze attention given by the Protection System for Women to children siblings of women victims of gender violence. Research findings indicate that professionals of police, social welfare and justice system believe that children are direct victims of gender violence and victims of psychological violence not only because of physical

\footnotetext{
* Save the Children. pepa@savethechildren.es
} 
Atención a los niños y las niñas víctimas de violencia de género

aggressions or as witness of aggressions, but because they experience violence in their diary home live. However, the research denounces also that the Protection System for Women has not created enough specific protection measures to cover children victims of gender violence, as well as their needs and participation in the decision making process for decisions going to concern them.

\section{KEY WORDS}

Children, Gender violence, Attention, Victims. 


\section{INTRODUCCIÓN}

Save the Children, como organización que trabaja en la defensa y promoción de los derechos del niño, ha venido desarrollando una línea programática en la prevención de violencia contra la infancia, desde las formas de castigo físico y psicológico hasta el abuso sexual infantil. En este contexto, Save the Children ha desarrollado una serie de demandas para garantizar, no sólo la prevención real y eficaz de las distintas formas de violencia contra los niños y niñas, sino para garantizar una atención psicológica, social, legal y sanitaria eficaz a aquellos niños y niñas involucrados en esta realidad, como víctimas o como agresores.

Paralelamente a este proceso, el trabajo de las organizaciones de mujeres, el aumento de la sensibilidad social y la concienciación de las instituciones ha generado una respuesta social única y en muchos sentidos modélica a un fenómeno que discurre paralelo al de la violencia contra los niños y niñas: la violencia de género. En los últimos años, tanto en el ámbito estatal así como en las distintas Comunidades Autónomas se han tomado las medidas específicas para afrontar un fenómeno cuya dimensión lo ha convertido en motivo justificado de alarma social.

Las medidas tomadas van en la misma línea de las demandas que Save the Children y otras organizaciones que trabajan en la erradicación de la violencia contra la infancia llevan años demandando: una legislación adecuada, protocolos de actuación conjunta que garanticen una intervención coordinada interdisciplinar e interinstitucional eficaz, la dotación de los recursos necesarios y suficientes, la profesionalización y formación específica de los y las profesionales responsables de la atención, la rapidez en los procedimientos y la prio- rización de la actuación cara a las víctimas.

Todo ello es para Save the Children motivo de esperanza, puesto que prueba que cuando la conciencia y la decisión política se unen, los cambios son posibles, tanto más cuando hablamos de niños y niñas cuya protección ha de ser responsabilidad prioritaria de las instituciones involucradas.

Sin embargo, el desarrollo de la Ley Orgánica 1/2004 de Medidas de Protección Integral contra la Violencia de Género y de los recursos que su aplicación y puesta en marcha ha conllevado ha generado una preocupación básica a la que esta investigación pretende dar voz: la atención a los niños, niñas y adolescentes hijos e hijas de mujeres víctimas de violencia de género.

Por todo ello, Save the Children desarrolló durante el año 2005 una investigación titulada "Atención a niños y niñas víctimas de la violencia de género: análisis de la atención en el sistema de protección a la mujer de los hijos e hijas de las mujeres víctimas de violencia de género". En este artículo se recogen los resultados básicos que arrojó dicha investigación.

\section{ALGUNAS CARACTERÍSTICAS QUE DEFINEN LA INVESTIGACIÓN DESARROLLADA}

No es ésta una investigación sobre sus madres sino sobre la atención a los niños y niñas y adolescentes hijos e hijas de mujeres víctimas de violencia de género dentro del sistema de protección de la mujer. No es tampoco una investigación sobre las distintas formas de violencia que viven los niños y niñas en el ámbito familiar, que podrían incluirse en la violencia doméstica, sino sobre los niños y 
niñas víctimas directas de la violencia de género. Tampoco es una investigación sobre el fenómeno de violencia de género en sí mismo, su magnitud ni su causalidad. El objetivo de esta investigación era analizar la intervención sobre el fenómeno desde el sistema de protección a la mujer, no el fenómeno en sí mismo.

Save the Children queria analizar si el sistema evalúa al menor como víctima de violencia de género, con todas las consecuencias que debería conllevar cara a dotación de recursos, creación de equipos y profesionalización de la intervención con estos niños y niñas entre otros.

Está por tanto fuera del objeto de esta investigación todas las mujeres y sus hijos e hijas que, aún siendo víctimas de violencia de género, no entran en contacto con el sistema y por tanto no podríamos constatar su existencia en un estudio como éste. Al centrar el estudio en la atención que se está dando a las víctimas, era fundamental limitarlo a la población sobre la que el sistema trabaja.

En este punto resulta significativo constatar que la conciencia de victima necesaria en mayor o menor medida, para entrar en contacto con el sistema, no ha de ser consonante entre las madres y los niños y niñas. Una mujer puede dar el paso y ser consciente de la violencia de su pareja y sus hijos e hijas no serlo, y viceversa, existen niños y niñas conscientes de ser víctimas de violencia de género pero que difícilmente pueden tomar medidas para salir de ello si sus madres no llegan a hacer consciente el problema y toman las medidas necesarias para ello.

Los objetivos de la investigación fueron los siguientes:

- Evaluar la percepción de las instituciones competentes y de los y las profesionales involucrados respecto a los niños y niñas hijos e hijas de las mujeres victimas de violencia de género como víctimas a su vez de esta violencia.

- Evaluar la atención que reciben los menores hijos e hijas de mujeres víctimas de la violencia de género en el sistema de protección a la mujer.

- Identificar los modelos de buena práctica ya existentes en la atención a los niños y niñas y realizar recomendaciones de mejora.

La metodologia de esta investigación fue cualitativa, basada en entrevistas a profesionales que atienden a estos niños y niñas y a sus madres y en recogida de información de las instituciones encargadas de su protección. Se realizaron $\mathbf{2 5 4}$ entrevistas personales y se recabó información adicional de 141 instituciones.

La investigación cuyos resultados se presentan se realizó con una metodología cualitativa en siete Comunidades Autónomas: Andalucia, Cataluña, Comunidad de Madrid, Comunidad Valenciana, Euskadi, Galicia y Islas Baleares, elegidas por los criterios de: incidencia del fenómeno, índice poblacional y estructura de Save the Children.

El equipo de investigación estuvo constituido por un grupo de investigadores autonómicos y una coordinadora estatal. Cada investigador autonómico desarrolló la investigación de campo, las entrevistas en base a protocolos de entrevista comunes y diferenciados por ámbitos a profesionales del ámbito social, policial y judicial y a mujeres en las casas de acogida, además de que en algunas comunidades autónomas se realizó una actividad de dibujo con niños y niñas en programas de atención psicoló- 
gica en las casas de acogida. Una vez finalizado, el equipo de investigación elaboró las conclusiones comunes y comparativas de las diferentes comunidades autónomas y la coordinadora estatal escribió el informe final estatal resultante de la investigación.

La investigación se realizó en el año 2005, y la recogida de datos desde Abril a Julio. Este dato es importante para contextualizar los resultados, dado que en el transcurso de la misma investigación, a veces incluso debido a ésta, en varias Comunidades Autónomas se tomaron medidas, y desarrollaron planes que no pudieron recogerse en la investigación escrita.

\section{RESULTADOS MÁS RELEVANTES}

\section{Magnitud del fenómeno}

El primer dato relevante de la investigación tuvo lugar cuando quisimos conocer cuántos niños y niñas vivían en un contexto de violencia de género y no pudimos hacerlo. El sistema recogia los datos sobre las mujeres, como victimas de la violencia de género, pero no sobre sus hijos e hijas, porque al no ser considerados víctimas de la violencia de género no son contabilizados a no ser que exista una orden de protección por agresión directa física contra ellos. De este modo, nos fue imposible obtener una idea de la magnitud del fenómeno de los niños y niñas victimas de la violencia de género, sencillamente porque no existen datos al respecto.

El único dato que obtuvimos se refiere al efecto más extremo de esta violencia de género sobre los niños y niñas que es su propia muerte ha sido a través de la Federación de mujeres Separadas y Divorciadas que hace un seguimiento en medios de comunicación de los casos de falleci- miento por violencia de género. En este seguimiento se encontraron 9 muertes de niños y niñas en 2004 y 7 en 2005 relacionadas con casos de violencia de género.

Es muy difícil desarrollar medidas adecuadas para atender a una población de la que se desconocen los datos fundamentales: cuántos son, dónde están y en qué circunstancias.

\section{Los efectos de la violencia de género sobre los niños y las niñas}

Los niños y niñas hijos e hijas de las mujeres victimas de la violencia de género son victimas directas de esta violencia y no han sido reconocidos como tales. Se les considera sólo víctimas directas cuando han sufrido las agresiones físicas conjuntamente con sus madres, y en el resto de los casos, se les considera a lo sumo victimas indirectas, en la medida que presencien estas agresiones a las madres, cuando no se argumenta que la violencia de un hombre a una mujer no cuestiona su capacidad como padre.

Los niños y niñas son víctimas de la violencia de género que se ejerce hacia sus madres no sólo, que también, porque a veces se les agreda también a ellos físicamente, siendo victimas de violencia física, sino porque siempre son víctimas de violencia psicológica y no sólo porque presencien las palizas o las agresiones a sus madres sino por el mero hecho de vivir en un entorno en donde esta violencia es una pauta de relación.

Viven en un entorno donde las relaciones violentas y el abuso de poder, que justifica, legitima y desencadena esa violencia como parte de las relaciones afectivas y personales son una pauta diaria. Internalizan un modelo negativo de relación que daña su desarrollo. Ven y sufren a una madre maltratada, en vez 
de protectora. Ven y sufren un padre maltratador, en vez de protector.

En la Ley Orgánica 1/2004 de Medidas de Protección Integral contra la Violencia de Género se habla del "síndrome de mujer maltratada" (en la exposición de motivos) , y se reconoce que estas situaciones "afectan también a los menores que se encuentran dentro de su entorno familiar ..." (Exposición de motivos II). La ley recoge además como agravante de la pena del agresor el hecho de que la agresión a la mujer se haya dado en presencia de los hijos e hijas. Pero estos son los dos únicos momentos donde la Ley atiende y refleja la realidad de estas víctimas de la violencia de género.

Save the Children considera que garantizando los derechos de las mujeres víctimas de violencia de género protegemos los de sus hijos e hijas, pero que hacen falta medidas adicionales especificas para atender sus necesidades.

En este sentido, los resultados básicos surgidos de las entrevistas con los profesionales fueron los siguientes:

- Todos los profesionales entrevistados reconocen que los niños y niñas son víctimas de la violencia de género, en todos los casos psicológica y a veces también física directa.

- El cuadro de síntomas que describen es similar al de un menor víctima de maltrato físico o emocional.

- Percepción generalizada del riesgo de que los niños repitan patrones de violencia vividos en su familia.

Entre los efectos de la violencia de género sobre los niños y niñas identificados por Save the Children y los profesionales entrevistados en esta investigación, destacamos los siguientes:
- Problemas de socialización: aislamiento social, dificultad para establecer relaciones personales, conductas agresivas o autoagresivas...

- Problemas escolares: bajo rendimiento, problemas de atención y concentración, agresiones entre iguales (como víctimas y como agresores)...

- Síntomas de estrés postraumático: trastornos del sueño, fobias, trastornos alimenticios, ansiedad, ataques de pánico...

- Conductas regresivas.

- Síntomas depresivos y de ansiedad.

- Alteraciones de desarrollo afectivo: modelos de vinculación erróneos, en los que violencia y amor van unidos, manejo del poder sobre otros...

- Internalización roles violentos o de género equivocados: cuidado con creer que los patrones de género se trasmiten por el sexo! Hay niñas que reproducen patrones de agresión y niños de víctima.

- Parentalización de los niños: asunción de roles adultos en un intento de proteger a sus madres...

Un criterio básico de la formación de los y las profesionales es hacerles ver que la violencia no es sólo la agresión física y la violencia de género es prueba de ello: no son las lesiones físicas sino el miedo y la anulación que sufren tanto mujeres como niños y niñas lo que los iguala en su condición de víctimas. La violencia no es sólo física. Crecer en un entorno de violencia daña el desarrollo de los niños y niñas aunque no sean golpeados directamente 0 presencien las palizas, porque crecen entendiendo la violencia como una pauta normal de relación. 
Para ejercer violencia es necesario que logre una situación de poder sobre esa persona. La violencia no es una cuestión de fuerza física, ni de sexo, sino de poder, de anular al otro hasta someterlo a mi voluntad. La mejor forma de lograr esto es a través de una combinación perfecta: elegir la víctima más vulnerable, la más débil, y establecer una relación afectiva con la víctima (una de las formas más claras de poder sobre otra persona es el amor) de forma que anule su capacidad de autonomía y su dignidad individual.

En el caso de la violencia de género, además, se perpetúan patrones de género destructivos y vinculados al abuso de poder.

Éstos fueron los presupuestos básicos de la investigación que pretendió valorar si el sistema percibe a estos niños y niñas en su condición de víctimas y qué tipo de atención les está proporcionando.

\section{Análisis de la atención a los niños y niñas por parte del sistema de protección a la mujer}

Respecto a las medidas que el sistema de protección a la mujer ha desarrollado para atender a los niños y niñas, encontramos dos conclusiones básicas:

\section{A. El sistema de protección a la mujer no contempla a los niños y niñas como victimas de la violen- cia de género, lo que posibilita su victimización secundaria.}

- No aparecen medidas específicas para atender a los menores en los documentos técnicos ni en los planes de acción.

- Existe una falta de coordinación entre los recursos del sistema de protección a la mujer y de protección infantil, que hace que el sistema de protección a la mujer tenga dentro de sus recursos niños y niñas que no están siendo atendidos por recursos que existen en el sistema de protección infantil y que podrian beneficiarles significativamente: recursos terapéuticos, educadores, trabajo con la escuela etc. No se realiza una notificación sistemática ni se cruzan los datos de ambos servicios.

- Los profesionales entrevistados argumentaron que la atención a los niños y niñas se garantizaba es a través de la madre, no desarrollando recursos específicos para sus necesidades. Encontramos escasos recursos terapéuticos o educativos específicos para los niños y niñas. Existen muy pocas comunidades como la Comunidad Valenciana 0 Cataluña donde haya educadores dentro de los centros de acogida para mujeres víctimas de violencia de género que den seguimiento a sus hijos e hijas, y muy pocos programas de tratamiento y apoyo psicológico para éstos.

\section{B. Los menores son tratados como} objeto de protección, no como sujetos de derecho:

- No se informa, acompaña o escucha a los niños y niñas en el proceso. El sistema judicial toma medidas que van a afectar la vida de estos menores sin evaluarlos en ningún momento. En este punto la rapidez requerida para las medidas cautelares por la ley dificulta cuando no imposibilita esta evaluación, pero habría que plantearse que medidas como el régimen de visitas no pueden tomarse en esas 72 horas y sin haber evaluado al núcleo familiar completo, incluido 
el niño. Además no existe un acompañamiento en el proceso a estos niños y niñas, que pueden verse saliendo de su casa y de su colegio, entrando un uno nuevo, obligados a guardar confidencialidad sobre el centro y la escuela ante sus amigos, familia e incluso con su padre en caso de haber régimen de visitas, $\mathrm{y}$ nadie les prepara para ello.

- Los niños varones mayores de doce años en muchos casos no acceden a los recursos residenciales preparados sólo para mujeres. Existen dos argumentos básicos para esta decisiôn: los problemas de conducta que presentan estos adolescente, en muchos casos repitiendo patrones agresivos, y por otro, la dificultad de convivencia para las mujeres víctima con estos menores en los que a menudo pueden proyectar y ver reflejada su propia historia.

- No existen recursos adaptados para mujeres y/o menores con alguna discapacidad, ni con trastornos mentales o con drogodependencias. En muchas ocasiones, se les deriva primeramente a servicios que atienden estas características en vez de a los centros de protección.

- No hay espacios físicos específicos para los menores en el ámbito policial y judicial. Los niños y niñas, sobre todo en el ámbito judicial, no disponen de espacios ni profesionales para atenderles, a menudo están esperando en el pasillo de un juzgado o de una comisaria mientras sus madres declaran. La falta de espacios físicos en el ámbito judicial para que agresor y víctimas no tengan que encontrarse en los momentos previos a los juicios es una de las demandas más básicas que hemos encontrado.

\section{CONCLUSIONES Y RECOMENDACIONES DE MEJORA}

El objetivo último del estudio de Save the Children no es sólo analizar la atención a los niños y niñas víctimas de la violencia de género, como realizar recomendaciones de mejora para las carencias que se detecten en el curso de la investigación.

Las recomendaciones resultantes de la investigación son las siguientes:

Recomendaciones de carácter general:

- Complementar la política actual de discriminación positiva de las mujeres con una politica garantista de los derechos de sus hijos e hijas.

- Promover estudios sobre la dimensión del problema, los efectos que la violencia de género sobre los niños y niñas y sus necesidades.

- Garantizar el cruce de los datos estadísticos entre los sistemas de protección a la mujer y a la infancia.

- Fortalecer la coordinación de los servicios a través de protocolos de actuación institucional establecidos y adecuadamente implementadas.

- Proporcionar la formación interdiscipinar a los profesionales del sistema de protección a la mujer sobre la atención a los niños y niñas.

- Promover las iniciativas y programas de buena práctica ya existentes incrementando su dotación presupuestaria y humana.

Recomendaciones sobre los recursos del ámbito social: 
- Reforzar los recursos de atención no residencial. Los profesionales insistían en reforzar la atención psicosocial a las víctimas además de la atención jurídica y policial.

- Promover e incrementar los programas de tratamiento terapéutico. Los centros deberian contar con educadores que acompañen, apoyen y supervisen específicamente a estos niños y niñas y con recursos de tratamiento terapéutico especializados, bien dentro del sistema de protección a la mujer, bien sea conveniados con el sistema de protección infantil.

- Incorporar la política de género al sistema de protección a la mujer, incluyendo educadores varones en los centros que proporcionen modelos masculinos positivos a los niños y niñas.

- Dotar de los mismos recursos a los servicios conveniados, concertados 0 propios de la administración

Recomendaciones sobre el acceso a los recursos:

- Garantizar el acceso a los adolescentes varones en los centros residenciales, preservando la unión de la madre y hermanos y dotando a estos centros de recursos de tratamiento especializados para los trastornos de conducta que éstos puedan presentar.

- Incrementar los escasos recursos adaptados para víctimas (mujeres y/o niños y niñas) con alguna discapacidad física, sensorial o psíquica.

- Crear recursos de tratamiento especializado para mujeres y niños y niñas con trastornos mentales y toxicomanias activas.
- Crear espacios físicos específicos para la atención a los niños y niñas en las sedes policiales y judiciales.

Sobre algunos aspectos del procedimiento judicial:

- Informar del mismo modo (amplitud, detalle y adaptado a su edad) a los niños y niñas que a las mujeres del proceso. El niño o niña han de saber lo que va a suceder y por qué para poder enfrentarlo.

- Dotar de equipos psicosociales especializados en los juzgados de actuación 24 horas que puedan trabajar con los niños y niñas y realizar las evaluaciones necesarias para la toma de decisiones que les afectan.

- La toma de declaración al menor como prueba preconstituida. Ésta es una medida de protección a los niños o niñas víctimas de violencia que debería establecerse de forma obligada y no ser de cumplimiento facultativo por parte del juez. La toma de declaración del menor por parte de un profesional formado y en unas condiciones protectoras no sólo impide la victimización secundaria sino que garantiza el pleno respeto a los derechos del niño como víctima, posibilitando las condiciones óptimas para su declaración.

- Incrementar, como se ha indicado, los espacios físicos adecuados para los niños y niñas en el ámbito policial y judicial.

- Suspender de forma sistemática y temporal las visitas de los hijos e hijas a sus padres en cuanto éstos hayan sido denunciados por situaciones de violencia de género para realizar una valoración de la unidad familiar. 
- Facilitar dispositivos diferenciados de los puntos de encuentro dependientes de los juzgados de violencia de género para la intervención en casos de violencia donde la mediación no es viable. Los puntos de encuentro fueron recursos creados para la mediación en casos de separación familiar y el cumplimiento del régimen de visitas, no para los casos de violencia en los que el niño o niña puede ser víctima de manipulación y presiones por ambos progenitores en este proceso sin ser adecuadamente detectado.

\section{Otras recomendaciones:}

- Los medios de comunicación juegan un papel fundamental en la información de cada caso y la generación de una conciencia social, dando una perspectiva constructiva del problema, sus vertientes y soluciones.
Save the Children considera que el momento actual, dado el desarrollo y aplicación de medidas legislativas, institucionales y sociales únicas, es óptimo para contribuir, tanto desde el conocimiento de los y las profesionales que trabajan con las víctimas como desde la vivencia de las propias mujeres y niños y niñas víctimas, a la consecución de los objetivos planteados por una ley tan joven como ciertamente esperanzadora.

Save the Children espera que las recomendaciones de mejora y modelos de buena práctica identificados en este estudio contribuyan a llamar la atención de la sociedad y las instituciones sobre una forma de violencia contra los niños y niñas que no siempre les deja huellas físicas pero que marca su presente, sus posibilidades de futuro y su modo de entender las relaciones afectivas.

\section{BIBLIOGRAFÍA}

Todos los informes están disponibles de forma integra a través de nuestra página web www.savethechildren.es

Save the Children (2006) "Atención a niños y niñas víctimas de la violencia de género" Informe estatal. Coord: Pepa Horno Goicoechea.

Save the Children (2006) "Atención a niños y niñas victimas de la violencia de género en Andalucia” Jose Angel Ponce.

Save the Children (2006) "Atención a niños y niñas víctimas de la violencia de género en Cataluña”. Asun Val Lisó, Amparo Cerezo Rincón, Lourdes Aramburu Otazu, Carme Calafat Siquier.

Save the Children (2006) "Atención a niños y niñas víctimas de la violencia de género en la Comunidad Valenciana”. Raquel Orejudo, Gema Gonzalez y Maria Perez Conchillo. Coord: Pepa Horno Goicoechea.
Save the Children (2006) "Atención a niños y niñas víctimas de la violencia de género en la Comunidad de Madrid". Dolores Perea y Rosa Maroto Coord: Pepa Horno Goicoechea.

Save the Children (2006) "Atención a niños y niñas víctimas de la violencia de género en Euskadi” Antonio J. Perdices, Coord: Pepa Horno Goicoechea.

Save the Children (2006) "Atención a niños y niñas víctimas de la violencia de género en las Islas Baleares". Juan Manuel Gil, Coord: Pepa Horno Goicoechea.

Save the Children (2005) "Atención a niños y niñas víctimas de la violencia de género en Galicia”. Santiago Miguez Caridad, IGAXES, Colegio de Trabajadores Sociales, Coord: Pepa Horno Goicoechea. 